\title{
Simulation of Deformation of the Adhesive Layer of the Spine of the Book Back of the Thread-Stitched Book Block
}

\author{
Petro KYRYCHOK*, Oleksandr PALIUKH** \\ *National Technical University of Ukraine, Igor Sikorsky Kyiv Polytechnic Institute, 37, Prosp. Peremohy, Kyiv, Ukraine, \\ E-mail: t_kyrychok@ukr.net \\ **National Technical University of Ukraine, Igor Sikorsky Kyiv Polytechnic Institute, 37, Prosp. Peremohy, Kyiv, \\ Ukraine, E-mail: alekspalyuh@gmail.com
}

cross $^{\text {ref }}$ http://dx.doi.org/10.5755/j01.mech.26.2.25854

\section{Introduction}

One of the factors that influence the long-term exploitation of book products, namely that part related to thread-stitched book block, is to ensure, first of all, reliable binding of the spine with the help of stitches of book thread, the use of adhesives of high tackiness, temperature resistance, high initial adhesion, high breaking load, which provide firmness of binding and resistance to plastic deformation caused by turning over the pages and notebooks of the block during reading, transportation, storage reservoirs, or other actions, which cause technological and operational damage to the spine of the book, and therefore the external presentation form and ease in obtaining information.

Based on the results of deformation simulation of the adhesive layer of the thread-stitched book block to determine peculiarities of load distribution of elastic deformation during bending of the adhesive spine plate while using the book products, to perform geometrical construction of the W-shaped adhesive plate, to calculate its area of cross-section and the amount of adhesive composition necessary and sufficient during the technological process of edging the book blocks, in order to minimize the spread of glue per unit area of the book spine.

The results of the conducted research will help to clarify and reduce the numerical indicators of the existing cost of adhesive compositions, which exist in the printing industry, comparing with the estimated ones, in order to save expensive book materials and, consequently, to reduce the costly economic component in calculating the cost for manufacturing book products and to improve their competitiveness.

The purpose of the work is to study and simulate the deformation of the adhesive layer of the spine of the thread-stitched book block, the nature of appearing of its damages and destruction caused by intensive use of the book while multiple turnover of pages and notebooks, as well as the influence of additional efforts aimed at opening of the block to the angles, close to $180^{\circ}$, which ensures comfortable reading.

\section{Experimental details}

It is known [1] that, by constructive manner, adhesive bonds, introduced in the processes of bookbinding production, are divided into flat, $\mathrm{W}$-shaped, $\mathrm{T}$-shaped and combined. Adhesive bonds of the W-type are used in the bookbinding processes of binding the spine of book blocks, which are previously thread-stitched for long-term preservation of the shape of the spineduring intensive exploitation of book products (Fig. 1).

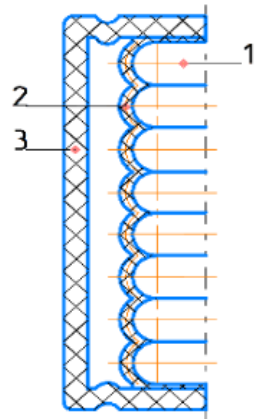

Fig. 1 Cross-section of the spine part of book block with 1 - book notebooks, 2 - W-type adhesive layer, 3 - cover

For studying and simulation of the deformation of the adhesive layer during bending of the spine of the book block (Fig. 2), there was made a selective choosing of the book blocks stitched by threads with the $\mathrm{W}$-like adhesive bond with different thickness of the adhesive layer. The selection of paper from which the book blocks were made is carried out from a wide range of paper type, with a smooth transition of their mass and thickness in samples.

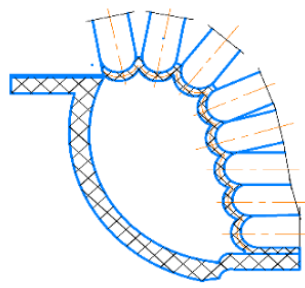

Fig. 2 Cross-section of the spine part the of a partially opened book block

Modern high-speed lines for production of books stitched by threads, the adhesive compositions, based on connecting properties, are spread by one technological layer on the spine of the book block to which the edge tape or a soft cover is adhered.

The recipe properties of modern adhesive compositions provide practical soaking of adhesive and wetting as by spine part of the surface of the book block as well as by paper tape, or adhering cover, and, accordingly, provide a layer of adhesive sufficient to fill the capillaries and 
pores of two adhering parts - the spine of the book block and edge paper tape [2].

As a result of technological operations of stitching of book blocks with threads, applying adhesive compositions to the spine part of blocks and adhering of edge tapes, forms an adhesive polymer plate between the spine of the block and the paper tape (Fig. 3), the thickness of which may vary within wide limits, determined by the formats of the edition, the number of notebooks in the book block, the number of pages in notebooks, the thickness and types of paper, etc.

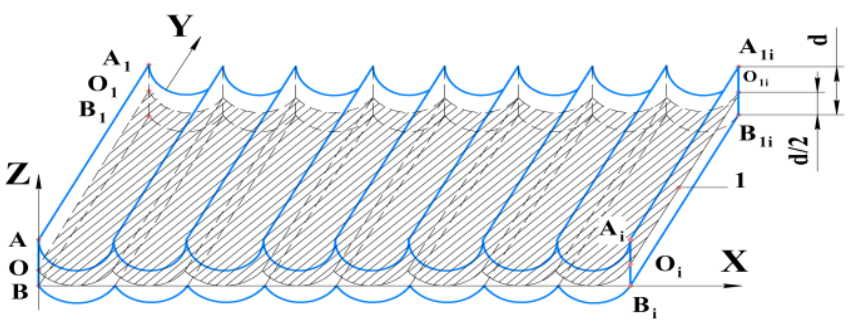

Fig. 3 Polymer plate formed by $S$ - shaped adhesive layer while head capping the book block, 1 - medium area, $d$ - thickness of the plate, $d / 2$ - distance to the middle plate

It is possible to assume that if adhesive polymeric plate where one (caved-in) side of the adhesive structure includes a certain plane formed by the binding of stitches of nylon or cotton threads, which were used for stitching the book blocks, and another (curved) side inserts an edge paper tape to the surface structure of adhesive, then such adhesive polymer plate is an elastic composite medium (adhesive composition) with inclusions from other materials.

A fragment of such an adhesive polymer composite plate reinforced with threads and a paper tape is given at Fig. 4, where vectors $\overrightarrow{P_{t n}}$ denote the direction of the tension forces of the threads implanted in the upper part of the adhesive layer, which, when the block is opened, are applied to the efforts of the tense state of the threads already formed in the process of stitching the block and its dense (pulling) of its spine, also vectors $\overrightarrow{P_{d s}}$ show the direction of the destruction efforts of the paper edge tape during multiple openings of the block and turning over of pages of the book.

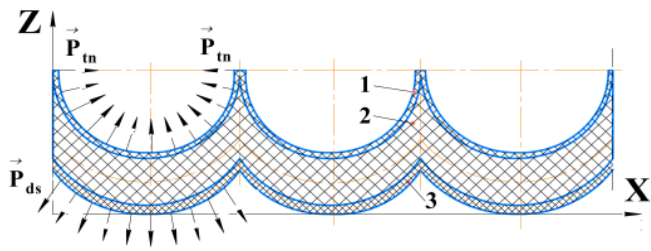

Fig. 4 Fragment of cross-section of the adhesive polymer plate reinforced by threads and paper tape: 1 - book threads, 2 - W-shaped adhesive layer, 3 - headcap paper tape, $\overrightarrow{P_{t n}}-$ vectors of tension efforts when opening the block, $\overrightarrow{P_{d s}}-$ vectors of efforts aimed at damager of the paper head cap tape when opening the block

Analyzing the processes of stitching the book blocks with threads, it should be noted that the magnitude of the regulation of the threaded system [3], on one hand, should ensure the maximum tension of the threads for dense bonding of the spine block, on the other, should not exceed the limit of effort that will lead to the destruction of openings formed by needle stabs in the spine part of notebooks, which may bring to partial sticking of adhesive through broken openings in the middle of notebooks during edging of the book block and spot adhering of the inner part of the spines of the folded notebooks of the book.

The term of using the adhesive polymer plates in the spine part of the book blocks primarily depends on the distribution of tensions that occur when the pages of the blocks [4] are turned over and, consequently, discrete bends of the adhesive polymer plate in the reading areas. The efforts that arise during formation of natural edges during opening of the block, when the pages of the book are cut off under their gravity, when turning over the pages, are also combined with the efforts applied to the opening of blocks at angles close to $180^{\circ}$ for comfortable reading.

The wavy geometrical construction of the W-like adhesive layer assumes the same, acceptable for simulating the deformation of the polymeric plate, thickness in the semi-circular areas of the spine tangent to folds of the book notebooks and increased thickness of the adhesive layer in places of formation of wedge-shaped tides with caved-in surfaces in the inter-spine space of notebooks, stitched by threads, proportionality of which (wedge-shaped flows) to semi-circular areas varies from 1:1.5 to 1:1.9.

Given the dependence on the nature of forces influencing the bends, the polymeric adhesive plates of the spine of the thread-stitched book block [5], may be referred to the plates working on the bend during the transverse load, and, given the nature of the load state of such plates, it is possible to refer them to the classification of flexible plates, and, while calculating thereof, it is necessary, in addition to bending loads, to take into account the loads, evenly distributed over the thickness (membrane stresses).

Despite of the inhomogeneous anisotropic structure of the adhesive composite of polymer plate, for convenience of simulating the deformation of the adhesive layer of the spine of the thread-stitched book block, it is possible to consider the adhesive plate as an isotropic body - homogeneous in structure in all directions, taking into account the coefficients of conformity, the numerical indices of which will be proportional to the types and materials from which the thread for stitching blocks are made, as well as the structure, firmness, density, mass, plasticity and other properties of paper, applied to edge blocks.

With the main operational factors [6] such as the stretching effort and the static and dynamic load influencing on the adhesive spine plate during reading, there may be a process of creation and development of cracks due to the effects of loads deferent in time, when each turning point formed during the page turning over and reading, can preserve the state of bending and stress, from short to long term, which is not proportional to the duration of action and place of effort application, along the entire adhesive polymer plate, leading to the heterogeneous, due to multiple factors, fatigue damage, determined by the sequence of occurrence of loads in discrete places of bending. During discrete opening of the book block in a numbered page sequence, or at any place of the stochastic sequence, there 
occurs the $\mathrm{W}$-like bending in the adhesive layer, during which, as can be observed during experimental studies [7], the bulk part of the adhesive layer close to the folds of book notebooks, is deformed in the form of elastic stretching, and the outer bulk part, close to the edge paper tape, is deformed in the form of elastic compression (Fig. 5, d).

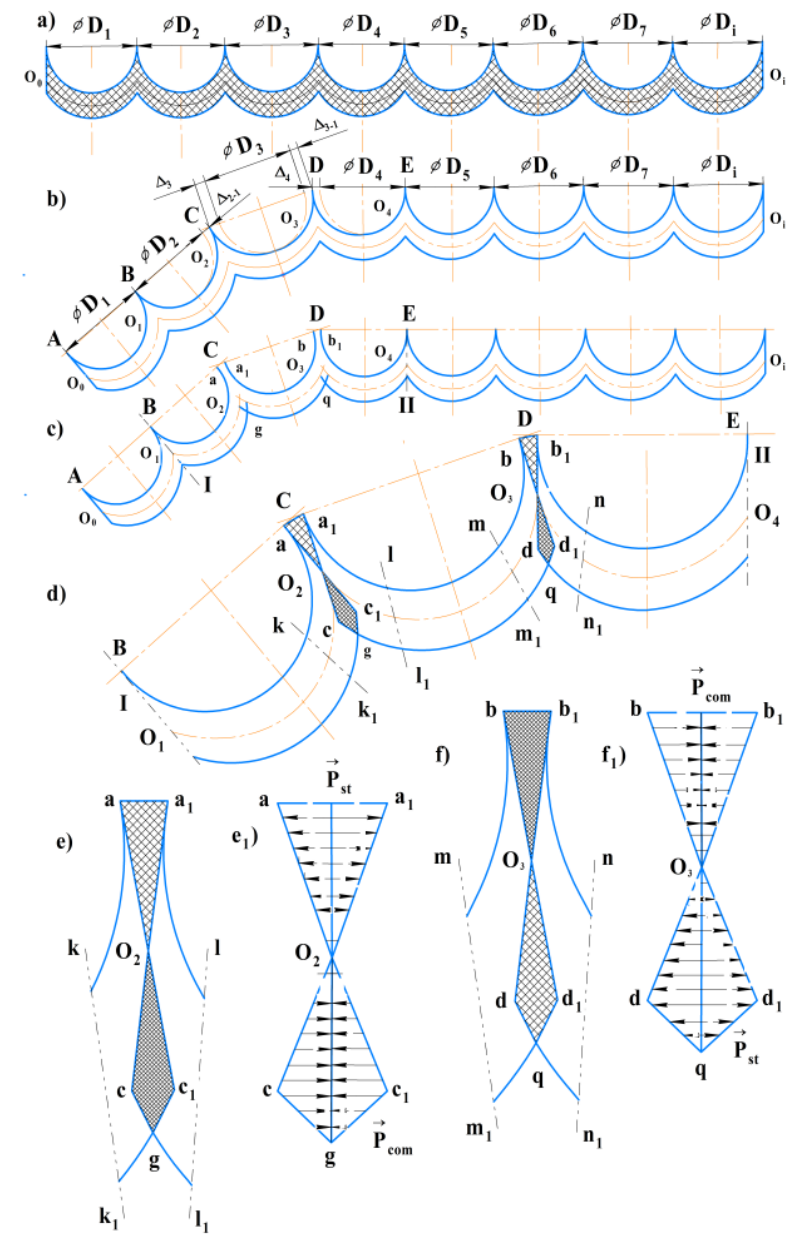

Fig. 5 Simulating the elastic and plastic deformation of Wshaped adhesive layer of the thread-stitched book block while turning over the pages and notebooks

During bending, in the stage of opening of the block, the plane of the upper layer (Fig. 3) of the polymeric adhesive plate, denoted by the contour $A A_{1} A_{\mathrm{i}} A_{1 \mathrm{i}}$ is tangent to the spine folds of the book notebooks (Fig. 2), which, during page turning over creates a load that deforms the top layer towards the elongation (changing the linear size towards increasement), and the plane of the lower layer, denoted by the contour $B B_{1} B_{\mathrm{i}} B_{1 \mathrm{i}}$, edge by the paper tape, does not change the linear size in the direction of reduction, but only accumulates compression load in the bounding places.

Based on the Kirchhoff's and Loves hypothesis for thin plates (hypothesis of inextensibility of the median plane) [8] is that the median plane of the plate does not deform during bending and that there are no deformations because of stretching, compression, and displacement in the median plane, it is adopted to simulate the assertion about the neutrality of the median plane of the adhesive polymeric plate of the spine of the thread-stitched book block, marked with the contour $O_{1} O_{\mathrm{i}} O_{1 \mathrm{i}}$ (Fig. 3), located at a distance of $d / 2$ from the outer surfaces of the adhesive plate ( $d$ is the thickness of the plate) and around which, as
0 (zero) mark, there may occur the diagrams of stretching and compression loads and places of bending of the plate (Fig. 5, $e_{1}, f_{1}$ ).

Upon finishing reading and returning the book to the original state in the folded form, the outer surfaces of the spine polymeric adhesive plate and the volumetric structures of these surfaces, located on both sides of the median plane, return to the original position without residual changes in the sizes that occur after multiple bends in the process of operational loads and transitions from the phenomena caused by elastic deformation of the adhesive plate to irreversible phenomena in demonstration of residual plastic deformation.

Fig. 5 shows the modeling of deformation of the adhesive layer of the root part of the book block sewn with threads: a) cross-section w-shaped layer of polymer glue by which the edging of the book block sewn with yarns is carried out; the book block is closed until pages are flipped; b) section of the adhesive layer at the beginning of the pages turning the book block and gradual bending of the root polymer plate; formation of stress zones under the action of tensile and compressive forces; c) cross-section of the adhesive layer depicting stretching and displacement zones formed by the forces of tension and compression of the wedge-shaped deltoid portion of the root plate; d) enlarged isolated section I-II of the adhesive layer depicting stretching and compression zones; e) increased area of stretching and contraction of the central part of the wedgeshaped deltoid section of the root plate; $\mathrm{e}_{1}$ ) vector group of efforts of stretching and compressing the central part of the wedge-shaped deltoid section of the root plate during the opening of the book block; f) enlarged separated section III of the adhesive layer depicting the areas of compression and stretching when closing the book block and alignment of the root plate; $f_{1}$ ) a vector group of efforts to compress and stretch a portion of the wedge-shaped deltoid portion of the root plate while closing the book block;

Fig. 5 shows the cross-section selected for simulation from the book block, W-shaped layer of polymeric adhesive, through which the edging of the thread-stitched book block is performed, without incorporating into the structure of the adhesive of threads used for stitching the block, as well as without an edge tape that is adhered to the spine of the block to increase the strength of the connection.

The caved-in parts of the cross-section of the adhesive polymer plate are formed by the rounding of the spines of the folded notebooks, from which the book block is formed. To simulate the bends of the adhesive plate, the geometric dimensions of the hemispheres are used, which form the adhesive plate on the upper and lower outer surfaces and correspond to the same thickness of book notebooks, the number of pages in which is constant, obtained by folding of paper sheets with the sizes determined by the format of the publication.

The number and size of identical notebooks in the unopened book block (Fig. 5 a) are indicated from the 1st and to the $i$-th, that is:

$$
\begin{aligned}
& \varnothing D_{1}=\varnothing D_{2}=\varnothing D_{3}=\varnothing D_{4}=\varnothing D_{5}= \\
& =\varnothing D_{6}=\varnothing D_{7}=\varnothing D_{i}=\text { const } .
\end{aligned}
$$


Line $O_{0} O_{\mathrm{i}}$, which divides the cross-section of the $\mathrm{W}$-like adhesive layer along the thickness in half, reflects the median plane (Fig.5, a, b, c).

When turning over the pages and gradually bending the spine of the polymeric adhesive plate (Fig. 5, b), the area of the correct semicircular shape of the plate, in the coordinates of the points $\mathrm{O}_{2}$ and $\mathrm{O}_{3}$ of the median line of the median plane, changes the configuration of the part, which adjoin to the spine of the folds of notebooks and change the linear dimensions $\left(S_{1}, S_{2}, S_{3} \ldots S_{i}\right)$ to some magnitudes $\Delta$, for example:

on the section $\mathrm{BC}$ for size $\varnothing D_{2}$ of the 2nd notebook in the book block, the change takes place towards the increase in size $\Delta_{2-1}\left(\varnothing D_{2}+\Delta_{2-1}=S_{2}\right)$;

- on the section CD for size $\varnothing D_{3}$ of the 3rd notebook in the book block, the change takes place towards the increase in size $\Delta_{3}$ and $\Delta_{3-1}\left(\varnothing D_{3}+\Delta_{3}+\Delta_{3-1}=S_{3}\right)$;

- on the section DE for size $\varnothing D_{4}$ of the 4 th notebook in the book block, the change takes place towards the increase in size $\Delta_{4}\left(\varnothing D_{4}+\Delta_{4}=S_{4}\right)$.

When turning over the pages of the block, from the beginning of the book to the end, the change in the geometric dimensions (identical in the folded book) of the section of the semicircular shape of the adhesive plate will occur during all the bends of the plate to the sizes that change in the direction of increasement, and the magnitudes $\Delta$ of size increasement, due to the elastic deformation of the plate, for each semicircular part, will be distinct, but not identical (only in some limited cases they may be equal), that is:

$$
\begin{aligned}
& \Delta_{1} \neq \Delta_{1-1} \neq \Delta_{2} \neq \Delta_{2-1} \neq \Delta_{3} \neq \\
& \neq \Delta_{3-1} \neq \Delta_{4} \ldots \neq \Delta_{i} \neq \text { const } .
\end{aligned}
$$

At each individual time $\Delta_{t}$, when reading a book and turning over pages, there is a change in the linear dimensions $\Delta_{S}$ of the sections of the adhesive plate, under the action of forces of elastic deformation.

Efforts that lead to a change in the linear dimensions of semicircular sections of the adhesive plate, during turning over the pages of the book, also affect the distribution of internal loads, from equilibrium, in the entire structure of the spine plate in a closed book, to formation of zones of stretching or compression that arise in places of discrete bending of the plate, especially in places of bending of the plate, which, approximately, are located between two notebooks (zones with centers $\mathrm{O}_{2}$ and $\mathrm{O}_{3}$ in Fig. 5, c).

On Fig. 5, d, conditional cuts $k k_{1}$ and $l l_{1}$ emphasize the part of the adhesive layer (section I-II), which demonstrates the zones of stretching and compression in the places of wedge-shaped spreading of adhesive and the formation of wedge-shaped deltoid configuration of the plate in the between-notebook sections of the spine.

When turning over the pages and applying additional efforts on the spine to obtain the opening angle of the block of up to $180^{\circ}$, in the zones marked at Fig. 5, d, as triangles $a a_{1} o_{2}$ and $b b_{1} o_{3}$, there is not only the accumulation of stretching forces of elastic deformation of the adhesive layer, which, when closing the book block will facilitate the return of the geometric parameters of the adhesive plate to their original position, but also the physical stretching in sections $\mathrm{aa}_{1}$ and $\mathrm{bb}_{1}$, which during long-term and repeated bending will lead to gradual destruction of the adhesive plate of the spine of the book block. Fig. 5, e shows the moment of bending of the adhesive plate during opening of the book block. Triangle $a o_{2} a_{1}$, shows the tensile forces, that have the maximum value at the vertex of the wedge-shaped part (section $a a_{1}$ ), are reduce and fall to zero at the point $o_{2}$ of the median line of the plate. Due to physical stretching of the adhesive material, the wedgeshaped part of the inter-spine zone (Fig. 5, b, at point C) turn into an inverse equilibrium triangle with vertex at point $\mathrm{O}_{2}$ and the base with the points $\mathrm{aa}_{1}$. At the same time, the bending of the plate, the deltoid part of the adhesive layer $o_{2} \mathrm{Cgc}_{1}$ accepts the compression forces maximally in section $\mathrm{cc}_{1}$, which lead to breaking of the adhesive layer in the zone with the end point $g$ and to the inner structural sealing and displacement of some sections in relation to the other (zones $c g$ and $c_{1} g$ ).

In the effort diagram (Fig. 5, $e_{1}$ ), the vector group $\overrightarrow{P_{s t}}$ of the stretching effort of the adhesive plate and the vector group $\overrightarrow{P_{c o m}}$ of the compression effort reproduce part of the elastic deformation processes that arise at the moment of discrete bending of the $\mathrm{W}$-like adhesive polymer plate in the tangent gap between the notebooks.

The reverse process of closing of the book block and returning of the spine adhesive plate to the original position, shown at Fig. 5, f, on which the conditional lines $\mathrm{mm}_{1}$ and $\mathrm{nn}_{1}$ (Fig. 5, d) highlight the part of the adhesive layer (section I-II), which reproduce zones of compression and stretching in places of wedge-shaped flows of adhesive with a vertex at point D (Fig. 5, b).

The pre-stretched part of the adhesive plate forming $\Delta b o_{3} b_{1}$ with accumulated elastic deformation forces returns to its original position when the block is closed, and the points $b$ and $b_{1}$ are aimed to their full merge at one vertex of the wedge-shaped between-notebook part of the spine adhesive plate, marked as vertex $D$ (Fig. 5, b), and the triangle $b o_{3} b_{1}$ transforms into a straight line. At the same time, the deltoid $o_{3} d q d_{1}$ begins stretching, due to the return of the elastically compressed inner structure of the adhesive plate to its original position. The points $d$ and $d_{1}$ aim to the lower vertex of the deltoid $q$, transforming the deltoid also into a straight line.

In the effort diagram (Fig. 5, $f_{1}$ ), the vector group $\overrightarrow{P_{c o m}}$ of the compression effort and the vector group $\overrightarrow{P_{s t}}$ of the stretching efforts of the adhesive plate, reproduce the part of the elastic deformation processes that arise when the spine of the adhesive plate is returned to its original position.

Long-term and numerous stretching of $\mathrm{aa}_{1}$ and $\mathrm{bb}_{1}$ of the adhesive plate, as well as compression of the internal structure of the plate in zones of $\operatorname{cgc}_{1}$ and $\mathrm{dqd}_{1}$ during the opening of the book blocks, and then the effect of alternating forces of elastic deformation before the return of the adhesive plate to its original position during the closure of the blocks, will contribute to the emergence of consequences, that are natural for residual plastic deformation of the elastic medium in the form of microcracks and irreversible destruction. While bending of the adhesive plate and the forming the zones of stretching and compression, not only do the place of the bends damage, but also the layer of adhesive elongates, which leads to an increase in 
the distance between the notebooks and the loss of firmness of the connection.

\section{Results}

In any particular case, for the book blocks stitched by threads, the studied distribution of the stretching and compression zones formed around the median line of the adhesive plate, is peculiar for any cross-section of the adhesive plate along the entire of the spine of the book.

To determine the numerical values of the cost of adhesive used for edging the spines of thread-stitched book blocks in order to minimize the cost of adhesive in practical use, let's consider the geometric construction of the adhesive polymer plate shown in Fig. 6, formed by concentric circles with radii corresponding to: $r$ is the rounded part of the spine of folded notebooks (notebooks of the same thickness with the same number of pages, $r=d_{n t}$, where $d_{n t}$ is the thickness of the notebook) and $R$ is the outer rounded part of the edged adhesive layer.

The sectional area of the $\mathrm{W}$-shaped adhesive plate consists of $n$ area of identical geometric fragments $S_{f r}$, denoted by section DCFE in Fig. 6, of the section $S_{0}$ and of the section $S_{i}$, in which, besides the geometric fragments of $S_{f r}$, the added sections $b_{0} c_{0} d_{0}$ and $d_{\mathrm{i}} c_{\mathrm{i}} b_{\mathrm{i}}$, which equal to $\frac{1}{2} S_{\text {segm }} a_{0} b_{0} c_{0} d_{0}$ and $\frac{1}{2} S_{\text {segm }} a_{\mathrm{i}} d_{\mathrm{i}} c_{\mathrm{i}} b_{\mathrm{i}}$ and are components of the side gap of the book block. Thus, the total area of the cross-section of the adhesive plate is:

$$
S_{\Sigma}=n S_{f r}+S_{0}+S_{i},
$$

where: $n$ is number of identical notebooks in the book block; $S_{f r}$ is the area of an individual fragment of the cross-section of the adhesive plate, which encircles the spine of one notebook; $S_{0}$ is the area of the cross-section of the adhesive edge of the first notebook; $S_{i}$ is the area of the cross-section of the adhesive edge of the i notebook.

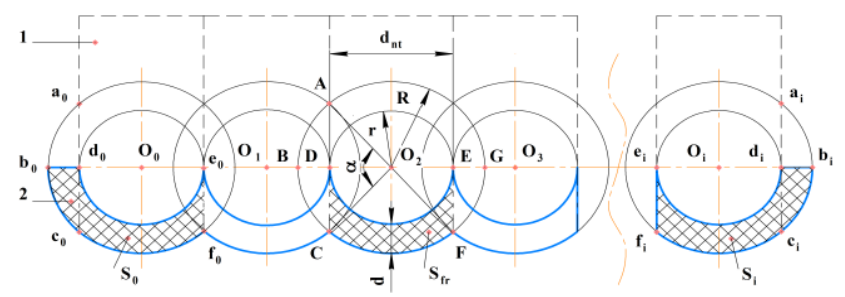

Fig. 6 Geometrical construction of the $\mathrm{W}$-shaped adhesive plate of the spine area of the book block for calculating the area of its cross-cut: 1 - spine fragments of thread-stitched book notebooks, $2-\mathrm{W}$-shaped adhesive layer, $d$-thickness of the adhesive layer, $d_{n}-$ thickness of the notebook

To determine $S_{f r}$ we will consider a ring with a center at the point $O_{2}$ in which the chord $A S$ is tangent to a circle with radius $r$, separates the $A B C D$ segment from the circle with radius $R$. Segments in the circle with radius $R$ are separated from both sides of the circle, forming a geometric figure denoted by a hatched zone DCFE obtained by separating the half-circle of $B C F G$ by $\frac{1}{2}$ of both segments marked as $B C D$ and $E F G$, that is:

$$
S_{f r}=S_{B C F G}-S_{B C D}-S_{E F G},
$$

where: $S_{B C F G}=\frac{1}{2} \pi\left(R^{2}-r^{2}\right) ; S_{B C D}=\frac{1}{4} R^{2}(\alpha-\sin \alpha)$; $S_{E F G}=\frac{1}{4} R^{2}(\alpha-\sin \alpha) ; \alpha$ is angle in radians.

$$
S_{f r}=\frac{1}{2} \pi\left(R^{2}-r^{2}\right)-\frac{1}{2} R^{2}(\alpha-\sin \alpha) .
$$

Respectively:

$$
\begin{aligned}
& S_{0}=\frac{1}{2} \pi\left(R^{2}-r^{2}\right)-\frac{1}{4} R^{2}(\alpha-\sin \alpha), \\
& S_{i}=\frac{1}{2} \pi\left(R^{2}-r^{2}\right)-\frac{1}{4} R^{2}(\alpha-\sin \alpha) .
\end{aligned}
$$

The amount of adhesive to edge the spine of the book blocks is determined as:

$$
K=S_{\Sigma} \cdot h \cdot \rho_{k} \cdot k_{y},
$$

where: $S_{\Sigma}$ is the area of the cross-section of the adhesive edge layer; $h$ is cut length of the book block; $\rho_{k}$ is density of adhesive during spreading the facing layer; $k_{y}$ is coefficient of shrinkage after drying the block.

The standards of adhesive consumption, for application in production, for edging the $\mathrm{W}$-like spine of the book block, taking into account the area of the spine block for 1000 copies, will be:

$$
H_{a d h}=1000 \frac{K}{S}\left(\mathrm{~g} / \mathrm{m}^{2}\right),
$$

where: $S$ is area of the spine of the book block.

For edging the book blocks on modern booklines of the 'Kolbus KM-600' type (Germany), there are foreseen standards of adhesive consumption (dispersion of polyvinyl acetate undiluted) for 1000 copies in the amount of $575 \mathrm{~g} / \mathrm{m}^{2}$ of the spine area of book blocks.

\section{Discussion}

The proposed calculation of the necessary and sufficient quantity of adhesive for the edged W-shaped spine of the book blocks, which considers the geometric features of such block bonding, provides the basic performance characteristics of using book products in firmness, wear resistance and durability, and creates conditions for minimizing the cost of adhesive used in technological process.

To determine the possibilities for minimizing the cost of adhesive, experimental studies have been carried out with conditions that ensure the application of the calculated adhesive layer during the production of circulating products at the 'Kolbus BF-512' line with circulation of more than 1 thousand copies.

Based on the circulating production using the stochastic method, there have been chosen samples to conduct the Flex Test on a Zigloh analytical device to determine the firmness and durability of the adhesive spine connection. Selected samples of circulating products, with the applied layers of adhesive, have been tested by Flex Test and shown data, which corresponded to the normative application of the adhesive on the spine of the thread-stitched block. 
The obtained average experimental values of the estimated amount of adhesive (polyvinyl acetate dispersion undiluted), which was applied to the spine part of the book block during the production of printing products, and normative indicators, for the most common formats of book products and the thickness of the spines of book blocks, are reproduced in the Table 1.

Based on the results of the tests, the amount of applied adhesive, calculated for 1thousand copies is
$500 \mathrm{~g} / \mathrm{m}^{2}$ of the spine area of the book blocks, which corresponds to $15 \%$ of adhesive composition savings, compared with the normative indicators $575 \mathrm{~g} / \mathrm{m}^{2}$ that are currently used in book production.

The experimentally obtained estimates of adhesive consumption are given in the table and are based on the technical and technological conditions of production, introduced in the largest book business in Ukraine - the State Publishing House 'Press Ukraine'.

Table 1

Average experimental values of the estimated amount of adhesive

\begin{tabular}{|c|c|c|c|c|c|c|c|c|c|c|}
\hline \multirow{4}{*}{$\begin{array}{l}\text { Thickness } \\
\text { of the } \\
\text { spine of } \\
\text { the book } \\
\text { block, mm }\end{array}$} & \multicolumn{10}{|c|}{ Format and length of the spine of the block } \\
\hline & \multicolumn{2}{|c|}{$\begin{array}{c}84 \times 108 / 32 \\
210 \mathrm{~mm}\end{array}$} & \multicolumn{2}{|c|}{$\begin{array}{c}84 \times 108 / 16 \\
270 \mathrm{~mm}\end{array}$} & \multicolumn{2}{|c|}{$\begin{array}{l}60 \times 84 / 8 \\
300 \mathrm{~mm} \\
\end{array}$} & \multicolumn{2}{|c|}{$\begin{array}{c}60 \times 90 / 16 \\
225 \mathrm{~mm}\end{array}$} & \multicolumn{2}{|c|}{$\begin{array}{c}70 \times 100 / 16 \\
250\end{array}$} \\
\hline & \multicolumn{10}{|c|}{ adhesive consumption for 1000 books, $g$} \\
\hline & 1 & 2 & 1 & 2 & 1 & 2 & 1 & 2 & 1 & 2 \\
\hline 10 & 1208 & 1050 & 1552 & 1350 & 1725 & 1500 & 1294 & 1125 & 1438 & 1250 \\
\hline 15 & 1811 & 1575 & 2329 & 2025 & 2587 & 2250 & 1941 & 1685 & 2156 & 1875 \\
\hline 20 & 2415 & 2100 & 3105 & 2700 & 3450 & 3000 & 2588 & 2250 & 2875 & 2500 \\
\hline 25 & 3019 & 2625 & 3881 & 3375 & 4312 & 3750 & 3234 & 2815 & 3594 & 3125 \\
\hline 30 & 3623 & 3150 & 4657 & 4050 & 5175 & 4500 & 3881 & 3375 & 4313 & 3750 \\
\hline 35 & 4226 & 3675 & 5433 & 4725 & 6037 & 5250 & 4528 & 3935 & 5031 & 4375 \\
\hline 40 & 4830 & 4200 & 6210 & 5400 & 6900 & 6000 & 5175 & 4500 & 5750 & 5000 \\
\hline
\end{tabular}

where: 1 - normative indicators of adhesive consumption; 2 - estimated adhesive consumption, introduced in production

\section{Conclusions}

Analysis of stages of deformation simulation of the binding adhesive layer of the thread-stitched book block has revealed process necessary for practical consideration such as stretching and compression of the book back adhesive polymeric plate.

The occurrence of damages and fractures of the plate during repeated turnover of pages of the book is caused by the alternative nature of the loads during its bending.

Except for the efforts that cause the formation of the natural opening angles of the book block there are also the efforts aimed at deformation of the spine to ensure comfortable reading.

According to the conditions and results of the studies, applying a calculated amount of glue to the book block spine, sewn with filaments, provides a long service life - identical to the book blocks in which the adhesive is applied, according to existing standards.

\section{References}

1. Vorobiev, D. V. 2007. Technology of postprint processes. Moscow: MGUP. 393 p. (in Russian).

2. Kulyk, L. Y. 2002. Improving the technology of edging the spine of book blocks with non-thread adhesive binding. Lviv: UAD. 24 p. (in Ukrainian).

3. Havenko, S. F.; Logaziak, I. Yu. 2012. The technology of striped fastening threads of book blocks. Lviv: UAD. 168 p. (in Ukrainian).

4. Petriashvili, G. G. 2007. Influence of the angle of opening of the book block on the result of evaluation of the strength of the adhesive compound by the pull test method. Book qualities: collection of scientific works. Lviv: UAD. Issue 2. p. 89-93.
5. Petriashvili, G. G. 2007. Mathematical model of the stress-strain state of the adhesive connection of the book block, Book qualities 1(45): 181-185.

6. Havenko, S. F.; Logaziak, I. Yu.; Turiab, L. V. 2012. Studying the factors that influence on the angles of opening of book blocks, Technology and technique of typography 1(35): 67-73 (in Ukrainian).

7. Paliukh, O. O. 2018. Experimental determination of the influence of book cover adhesives on the formation of natural angles of opening of book blocks, Technology and technique of typography 1(59): 37-47 (in Ukrainian).

8. Love, A. E. H. A treatisse on the matematical theory of elasticity. Translation from English-M.-L.: ONTI,1935. (in Russian).

\section{P. Kyrychok, O. Paliukh}

\section{SIMULATION OF DEFORMATION OF THE ADHE- SIVE LAYER OF THE SPINE OF THE BOOK BACK OF THE THREAD-STITCHED BOOK BLOCK}

\section{S u m m a r y}

The proposed calculation of the amount of adhesive composition, to envelop the W-like spine of the book blocks takes into account their geometric features and creates conditions for minimizing the glue spread in the technological process.

Keywords: block, spine, opening, edge, adhesive composition, plate bending, polymer deformation. 